\title{
Self-initiated physical activity is associated with high sensitivity C-reactive protein: A longitudinal study in 5,030 adults
}

\author{
Rômulo Araújo Fernandes a, Raphael Mendes Ritti-Dias b, *, P. Babu Balagopal c, \\ Raquel D.O. Conceição ${ }^{\mathrm{d}}$, Raul D. Santos ${ }^{\mathrm{d}, \mathrm{e}}$, Gabriel Grizzo Cucato ${ }^{\mathrm{d}}$, \\ Márcio Sommer Bittencourt ${ }^{\mathrm{d}, \mathrm{f}}$ \\ a Sao Paulo State University (UNESP), Presidente Prudente, Brazil \\ ${ }^{\mathrm{b}}$ Universidade Nove de Julho, São Paulo, Brazil \\ c Nemours Children's Specialty Care and Mayo Clinic College of Medicine, Jacksonville, FL, USA \\ ${ }^{\mathrm{d}}$ Hospital Israelita Albert Einstein, Sao Paulo, Brazil \\ e Lipid Clinic Heart Institute (InCor), University of São Paulo, Sao Paulo, Brazil \\ ${ }^{\mathrm{f}}$ Faculdade Israelita de Ciências da Saúde Albert Einstein, Sao Paulo, Brazil
}

\section{A R T I C L E I N F O}

\section{Article history:}

Received 4 October 2017

Received in revised form

23 January 2018

Accepted 7 February 2018

Available online 8 February 2018

\section{Keywords:}

Exercise

Body mass index

Cardiovascular risk

$\mathrm{C}$ reactive protein

Inflammation

\begin{abstract}
A B S T R A C T
Background and aims: Structured regular exercise programs decrease high-sensitivity C-reactive protein (hsCRP), a marker of low-grade inflammation in adults. Longitudinal effects of self-initiated physical activity levels (PAL) on hsCRP are less clear. This study evaluated the association of longitudinal changes in hsCRP in relation to modifications in PAL, over time, in a large sample of adults.

Methods: Participants included 5030 adults, 4045 (80\%) males, undergoing routine health screening examinations. Elevated level of hsCRP was defined as $\geq 3 \mathrm{mg} / \mathrm{L}$. Self-reported PAL, height, weight, blood pressure and blood samples were collected at baseline and after a median of 2.9 years (P25 1.97 and P75 $\left.^{\text {th }} 4.37 \mathrm{yrs}\right)$. Participants were stratified according to their PAL at baseline and follow-up as: i) persistently physically inactive; ii) became physically inactive; iii) became physically active; iv) persistently physically active (active both at baseline and follow-up).

Results: Persistently physically active participants had lower odds of having higher hsCRP (OR $=0.35$ [95\% CI: 0.25 to 0.48 ]). The maintenance of high PAL was associated with lower hsCRP in both sexes (men: $\mathrm{OR}=0.44$ [0.30 to 0.65$]$ and women: $\mathrm{OR}=0.35$ [0.16 to 0.76]). Participants with overweight/ obesity $(\mathrm{OR}=0.43$ [95\% CI: 0.29 to 0.63$])$ and smokers $(\mathrm{OR}=0.123$ [95\% CI: 0.03 to 0.60$]$ ) who were persistently active had lower odds of having higher hsCRP compared to physically inactive peers. Conclusions: Self-initiated PAL was longitudinally associated with hsCRP in adults. The data suggest that the initiation or maintenance of PA attenuates the low-grade inflammatory state, independent of sex, body weight and smoking status.
\end{abstract}

(c) 2018 Elsevier B.V. All rights reserved.

\section{Introduction}

Low-grade inflammation plays an important role in all stages of atherosclerotic cardiovascular disease and is associated with an increased risk of cardiovascular morbidity and mortality. C-reactive protein (hsCRP) is a non-specific marker of inflammation and is an independent biomarker of higher cardiovascular risk [1] and early mortality in adults [2]. Recently, the Canakinumab

\footnotetext{
* Corresponding author. Rua Vergueiro, 325. Morumbi, Sao Paulo, Brazil

E-mail address: raphaelritti@gmail.com (R.M. Ritti-Dias).
}

Antiinflammatory Thrombosis Outcome Study (CANTOS) trial showed that reduction in inflammation by a monoclonal antibody against interleukin 1-beta, and assessed by lowering of plasma hsCRP concentrations, was associated with lower rates of recurrent cardiovascular events, independent of LDL cholesterol levels [3].

Previous studies have shown that structured regular exercise programs decrease the circulating levels of various inflammatory biomarkers including hsCRP in adults and the elderly [4-6]. However, these exercise programs were usually of short duration and typically performed in research settings. Effective implementation of these intervention programs in real-life public health conditions is challenging. Previously we have demonstrated that 
self-reported changes in physical activity levels (PAL) were associated with improvements in several health parameters [7-9]. However, the potential longitudinal effect of unstructured and selfinitiated PAL, in free-living conditions, on residual hsCRP is unknown. Therefore, given the importance of hsCRP as a marker of higher cardiovascular risk, would be interesting to investigate whether individuals who maintain or increase their PAL present lower values of hSCRP in a longitudinal design study. Thus, the aim of this study was to identify the modifications in PAL over time and changes in hsCRP values in a large sample of adults asymptomatic for cardiovascular disease.

\section{Materials and methods}

\subsection{Study design}

We included all consecutive adults between 18 and 60 years of age, who participated in an employer-sponsored routine health evaluation at the Preventive Medicine Center of the Hospital Israelita Albert Einstein (Sao Paulo, Brazil) from January 2007 to December 2013. In this survey were considered only participants who returned for a follow-up visit at least one year after the initial encounter (for participants with multiple return visits, the last visit was used as the follow-up). All participants were invited to voluntarily participate in a health promotion program. We analyzed hsCRP levels and PAL at baseline and during a follow-up visit that occurred between 12 and 82 months after the initial encounter. The present study was approved by our institutional review board, and a consent form was waived.

Initially, the dataset consisted of 7741 individuals of both sexes who had baseline and follow-up data. To avoid misclassification of hsCRP values due to potential influence of acute inflammation, participants who had hsCPR levels $\geq 10 \mathrm{mg} / \mathrm{L}$ in any of the moments were excluded $(\mathrm{n}=311)$. Additionally, considering all variables involved in this study, individuals with any missing data $(\mathrm{n}=2169)$ and less than 12 months of follow-up $(n=231)$ were also excluded. Therefore, the final sample involved in this study was composed of 5030 adults (4045 men) that presented the complete data at baseline and follow-up.

Measurement of hsCRP was performed at baseline and followup visits. Blood samples were collected after an overnight fast and analyzed as part of the routine clinical workflow. Laboratory analyses included determination of hemoglobin concentrations, a standard lipid panel and fasting glucose using a Vitros platform automated laboratory system (Johnson \& Johnson Clinical Diagnostics, New Brunswick). The laboratory responsible for all blood analyses meets the standardized criteria for quality control adopted by the Brazilian Health Ministry. Measurement of hsCRP (expressed in $\mathrm{mg} / \mathrm{L}$ ) was performed by a turbidimetric method on a nephelometry system (Dade-Boehring, EUA).

PAL was assessed by the short version of International Physical Activity Questionnaire (IPAQ-SF) which was previously validated in a similar population [10]. The participants fulfilled the questionnaire under supervision of a trained staff member. At baseline, participants were classified according to the IPAQ-SF into one of two distinct qualitative categories of PA: physically active ( $\geq 150 \mathrm{~min} /$ week of moderate-to-vigorous intensity) or inactive ( $<150 \mathrm{~min} /$ week of moderate-to-vigorous intensity). Considering both baseline and follow-up, participants were classified into three categories: i) persistently physically inactive (inactive both at baseline and follow-up); ii) physically active in one time point (active at baseline or at follow-up); iii) persistently physically active (active both at baseline and follow-up).

Anthropometric assessments included measurement of body mass in $\mathrm{kg}$ and height in meters $(\mathrm{m})$. Body mass index (BMI [expressed as $\mathrm{kg} / \mathrm{m}^{2}$ ]) was defined as the individual's weight ( $\mathrm{kg}$ ) divided by the square of their height $\left(\mathrm{m}^{2}\right)$. Overweight/obesity was determined as BMI $\geq 25 \mathrm{~kg} / \mathrm{m}^{2}$.

Blood pressure was measured in triplicate, in the seated position, according to the standard method recommended by the American Heart Association [11] after the participants had rested for at least $5 \mathrm{~min}$. The measurements were performed in both upper limbs using the auscultatory method with an aneroid sphygmomanometer, and employing phases I and V of the Korotkoff sounds.

Medical records were accessed in order to identify the prescription of statins, as well as the previous diagnoses of arterial hypertension, dyslipidemia and diabetes mellitus. Alcohol consumption was assessed through face-to-face interview using the Alcohol Use Disorders Identification Test (AUDIT) [12].

\subsection{Statistical analysis}

Descriptive statistics for continuous variables were expressed as means and standard deviations, except for hs-CRP expressed in median and interquartile range. Categorical variables were presented as rates and their 95\% confidence intervals (95\% CI).

Baseline values of age, sex, blood glucose, hemoglobin, statin use, alcohol consumption (AUDIT), systolic and diastolic blood pressure (SBP and DBP, respectively) were used as covariates in the multivariate models. The time of follow-up was stratified into $1.0-1.9$ years, $2.0-2.9$ years and $\geq 3$ years. Independent variables were measured at two-time points (baseline and follow-up) and thus variables with longitudinal approach were created. For overweight/obesity three categories were created: BMI $\geq 25 \mathrm{~kg} / \mathrm{m}^{2}$ in none, BMI $\geq 25 \mathrm{~kg} / \mathrm{m}^{2}$ in just either baseline or follow-up and BMI $\geq 25 \mathrm{~kg} / \mathrm{m}^{2}$ in both baseline and follow-up visits. For smoking three categories were created: non-smoker in both visits, current smoker in just either baseline or follow-up and current smoker in both baseline and follow-up. Considering each disease diagnosed by the attending physician (hypertension, dyslipidemia and diabetes mellitus) three categories were created: positive diagnosis in none, positive diagnosis in just either baseline or follow-up and positive diagnosis in both baseline and follow-up. The comparison of sample characteristics among these groups was performed by ANOVA one-way, except for hs-CRP in which was used Kruskal-Wallis test.

A hsCRP value of $\geq 3 \mathrm{mg} / \mathrm{L}$ was defined as "higher hsCRP" [13]. The main outcome of this study was higher hsCRP in both baseline and follow-up. The impact of physical activity on hsCRP levels was estimated by binary logistic regression, which was expressed as odds ratios (OR) and 95\% CI. Multivariate models were developed adjusting for age, sex, arterial hypertension, dyslipidemia, diabetes mellitus, time of follow-up, smoking status, overweight/obesity, blood pressure, glucose, statin use, hemoglobin values and alcohol consumption (AUDIT). All statistical analyses were performed in the software BioEstat (release 5.0) and statistical significance ( $p$ value) was set at 0.05 .

\section{Results}

Table 1 shows clinical and laboratory characteristics of study participants. Mean age was $41.3 \pm 8.5$ and there was a higher proportion of men $(p$-value $=0.002)$. The median follow-up time was 2.9 years ( $\mathrm{P} 25^{\text {th }} 1.97$ and $\left.\mathrm{P} 75^{\text {th }} 4.37\right)$, the medians ( $\mathrm{P} 25^{\mathrm{th}}$ and $\mathrm{P} 75^{\mathrm{th}}$ ) for hsCRP were 1.1 (0.5 and 2.1) and 1.0 (0.5 and 2.0) at baseline and follow-up, respectively. The prevalence of higher hsCRP was similar over time (baseline: $14.8 \%$ and follow-up: $15.5 \% ; p=0.263$ ), while high levels of hsCRP at both baseline and follow-up were encountered in $6.5 \%$ (95\% CI: 5.8 to 7.2 ) of the entire sample.

Table 2 shows sample characteristics according to PAL groups. 
Table 1

General characteristics of the studied population $(n=5030)$.

\begin{tabular}{|c|c|c|}
\hline Variable & Categories & $\begin{array}{l}\text { Descriptive statistics } \\
n(\%)\end{array}$ \\
\hline \multicolumn{3}{|l|}{ Sex } \\
\hline & Male & $4045(80.4)$ \\
\hline & Female & $985(19.6)$ \\
\hline \multicolumn{3}{|c|}{ Age baseline } \\
\hline & $<30$ years & $366(7.3)$ \\
\hline & 30-39 years & $2018(40.1)$ \\
\hline & $40-49$ years & $1847(36.7)$ \\
\hline & $\geq 50$ years & 799 (15.9) \\
\hline \multicolumn{3}{|c|}{ Time of follow-up } \\
\hline & $1.0-1.9$ years & $1330(26.4)$ \\
\hline & $2.0-2.9$ years & $1338(26.6)$ \\
\hline & $\geq 3$ years & $2362(47.0)$ \\
\hline \multicolumn{3}{|c|}{ Body mass index $\geq 25 \mathrm{~kg} / \mathrm{m}^{2}$} \\
\hline & None & $1669(33.2)$ \\
\hline & Either baseline or follow-up & $607(12.1)$ \\
\hline & Both & $2754(54.7)$ \\
\hline \multicolumn{3}{|c|}{ Hypertension diagnosis (yes) } \\
\hline & None & $4229(84.1)$ \\
\hline & Only at follow-up & $267(5.3)$ \\
\hline & Both & $534(10.6)$ \\
\hline \multicolumn{3}{|c|}{ Diabetes diagnosis (yes) } \\
\hline & None & $4882(97.1)$ \\
\hline & Only at follow-up & $45(0.9)$ \\
\hline & Both & $103(2.0)$ \\
\hline \multicolumn{3}{|c|}{ Dyslipidemia diagnosis (yes) } \\
\hline & None & $2096(41.7)$ \\
\hline & Only at follow-up & $1428(28.4)$ \\
\hline & Both & $1506(29.9)$ \\
\hline \multicolumn{3}{|c|}{ Statin use } \\
\hline & None & $4108(81.7)$ \\
\hline & Either baseline or follow-up & $573(11.4)$ \\
\hline & Both & $349(6.9)$ \\
\hline \multicolumn{3}{|c|}{ Smoking (yes) } \\
\hline & None & $4453(88.5)$ \\
\hline & Either baseline or follow-up & $298(5.9)$ \\
\hline & Both & $279(5.5)$ \\
\hline \multicolumn{3}{|c|}{ C-reactive protein ( $\geq 3 \mathrm{mg} / \mathrm{L}$ ) } \\
\hline & None & $3833(76.2)$ \\
\hline & Either at baseline or follow-up & $870(17.3)$ \\
\hline & Both & $327(6.5)$ \\
\hline
\end{tabular}

Subjects physically active in both baseline and follow-up were older, presented a higher proportion of men, a lower proportion of smokers and diabetics, had lower BMI and hs-CRP concentrations $(p<0.05)$. Subjects that were physically active in one time point had lower hs-CRP concentrations and lower proportion of diabetics compared to the group that was not physically active at any time point $(p<0.05)$.

Table 3 shows univariate clinical associations with higher hsCRP at baseline and follow-up. In both assessments, higher hsCRP values
Table 3

Parameters associated with high hsCRP levels in study participants at baseline and follow-up $(n=5030)$.

\begin{tabular}{|c|c|c|c|}
\hline Variable & Categories & $\begin{array}{l}\text { High hsCRP } \\
\text { n (\%) }\end{array}$ & $p$ \\
\hline \multirow[t]{2}{*}{ Sex } & Male & $241(6.0)$ & 0.002 \\
\hline & Female & $86(8.7)$ & \\
\hline \multirow{4}{*}{ Age baseline } & $<30$ years & $33(9.0)$ & 0.223 \\
\hline & $30-39$ years & $107(5.3)$ & \\
\hline & $40-49$ years & $123(6.7)$ & \\
\hline & $\geq 50$ years & $64(8.0)$ & \\
\hline \multirow[t]{3}{*}{ Time of follow-up } & $1.0-1.9$ year & $108(8.1)$ & 0.023 \\
\hline & 2.0-2.9 years & $77(5.8)$ & \\
\hline & $\geq 3$ years & $142(6.0)$ & \\
\hline \multirow{3}{*}{$\mathrm{BMI} \geq 25 \mathrm{~kg} / \mathrm{m}^{2}$} & None & $51(3.1)$ & 0.001 \\
\hline & Either baseline or follow-up & $25(4.1)$ & \\
\hline & Both & $251(9.1)$ & \\
\hline \multirow[t]{3}{*}{ Hypertension diagnosis } & None & $259(6.1)$ & 0.0002 \\
\hline & Only follow-up & $14(5.2)$ & \\
\hline & Both & $54(10.1)$ & \\
\hline \multirow{3}{*}{ DM diagnosis } & None & $311(6.4)$ & 0.025 \\
\hline & Only follow-up & $4(8.9)$ & \\
\hline & Both & $12(11.7)$ & \\
\hline \multirow{3}{*}{ DLP diagnosis } & None & $122(5.8)$ & 0.210 \\
\hline & Only follow-up & $103(7.2)$ & \\
\hline & Both & $12(11.7)$ & \\
\hline \multirow[t]{3}{*}{ Statin use } & None & $274(6.7)$ & 0.097 \\
\hline & Either baseline or follow-up & $40(7.0)$ & \\
\hline & Both & $13(3.7)$ & \\
\hline \multirow[t]{3}{*}{ Smoking (yes) } & None & $269(6.0)$ & 0.001 \\
\hline & Either baseline or follow-up & $23(7.7)$ & \\
\hline & Both & $35(12.5)$ & \\
\hline \multirow[t]{3}{*}{ Physically active } & None & $182(9.0)$ & 0.001 \\
\hline & Either baseline or follow-up & $100(6.1)$ & \\
\hline & Both & $45(3.3)$ & \\
\hline
\end{tabular}

$\mathrm{BMI}=$ body mass index; $\mathrm{AH}=$ arterial hypertension; $\mathrm{DM}=$ diabetes mellitus; $\mathrm{DLP}=$ dyslipidemia.

${ }^{\text {a }}$ C-reactive protein $\geq 3 \mathrm{mg} / \mathrm{L}$ in both (baseline and follow-up).

were associated with female sex $(p=0.002)$, with shorter followup time $(p=0.023)$, overweight/obesity $(p=0.001)$, hypertension $(p=0.002)$ and diabetes at baseline $(p=0.025)$, and smoking $(p=0.001)$.

In the overall sample, $27 \%$ of participants were active at both time-points, $40.2 \%$ sedentary at both time-points, $20.7 \%$ became active from baseline to follow-up and $12.1 \%$ became sedentary from baseline to follow-up.

Persistently physically active participants both at baseline and follow-up had lower prevalence of higher hsCRP than persistently physically inactive counterparts (Active: $3.3 \%$ vs. Inactive: $9.0 \% ; p=$ 0.001).

Table 4 shows the multivariate adjusted prevalence of higher hsCRP at baseline and follow-up. The maintenance of high PAL

Table 2

Characteristics of the sample according to physical activity pattern $(n=5030)$.

\begin{tabular}{|c|c|c|c|c|}
\hline & \multicolumn{4}{|c|}{ Physically active (considering baseline and follow-up) } \\
\hline & None $(\mathrm{n}=2021)$ & Either baseline or follow-up $(n=1656)$ & Both $(\mathrm{n}=1358)$ & $p$ \\
\hline Sex (\% men) & 76.9 & $82.3^{\mathrm{b}}$ & $83.4^{\mathrm{b}}$ & $<0.01$ \\
\hline Age (years) & $40.8(8.2)$ & $40.9(8.6)$ & $42.7(8.8)^{\mathrm{b} c}$ & $<0.01$ \\
\hline C-reactive protein $(\mathrm{mg} / \mathrm{L})^{\mathrm{a}}$ & $1.20(1.80)$ & $1.10(1.50)^{\mathrm{b}}$ & $0.90(1.30)^{\mathrm{b}}$ & $<0.01$ \\
\hline Body mass index $\left(\mathrm{kg} / \mathrm{m}^{2}\right)$ & $26.4(4.1)$ & $26.2(3.7)$ & $25.6(3.3)^{b c}$ & $<0.01$ \\
\hline Smoking (\%) & 10.5 & 9.1 & $7.8^{\mathrm{b}}$ & 0.03 \\
\hline Hypertension (\%) & 10.9 & 10.1 & 10.8 & 0.71 \\
\hline Diabetes (\%) & 2.8 & $1.6^{\mathrm{b}}$ & $1.5^{\mathrm{b}}$ & 0.01 \\
\hline Dyslipidemia (\%) & 29.0 & 30.9 & 30.2 & 0.44 \\
\hline
\end{tabular}

\footnotetext{
a Expressed as median (interquartile range).

b Significantly different from none group.

c Significantly different from Either baseline or follow-up group.
} 
Table 4

Multivariate association of high hsCRP by physical activity level according to sex, age and time of follow-up ( $n=5030)$.

\begin{tabular}{|c|c|c|c|}
\hline \multirow[t]{3}{*}{ Variables } & \multicolumn{3}{|c|}{ Physically active (considering baseline and follow-up) } \\
\hline & \multirow{2}{*}{ None } & \multirow{2}{*}{$\frac{\text { Either baseline or follow-up }}{\text { OR }(95 \% \mathrm{CI})}$} & \multirow{2}{*}{$\frac{\text { Both }}{\text { OR }(95 \% \mathrm{CI})}$} \\
\hline & & & \\
\hline \multicolumn{4}{|l|}{$\operatorname{Sex}^{\mathrm{a}}$} \\
\hline Male $(\mathrm{n}=4045)$ & 1.00 (reference) & $0.677(0.497$ to 0.921$)$ & $0.429(0.290$ to 0.633$)$ \\
\hline Female $(\mathrm{n}=985)$ & 1.00 (reference) & $0.981(0.586-1.643)$ & $0.403(0.183$ to 0.884$)$ \\
\hline \multicolumn{4}{|l|}{ Age baseline ${ }^{\mathrm{b}}$} \\
\hline$<30$ years $(\mathrm{n}=366)$ & 1.00 (reference) & $1.323(0.541-3.231)$ & $0.231(0.043-1.234)$ \\
\hline $30-39$ years $(n=2018)$ & 1.00 (reference) & $0.793(0.508-1.238)$ & $0.541(0.298$ to 0.981$)$ \\
\hline $40-49$ years $(n=1847)$ & 1.00 (reference) & $0.579(0.366$ to 0.917$)$ & $0.390(0.224$ to 0.680$)$ \\
\hline$\geq 50$ years $(n=799)$ & 1.00 (reference) & $0.606(0.309-1.188)$ & $0.334(0.152$ to 0.739$)$ \\
\hline \multicolumn{4}{|l|}{ Time of follow-up ${ }^{c}$} \\
\hline $1.0-1.9$ year $(\mathrm{n}=1330)$ & 1.00 (reference) & $1.022(0.641-1.629)$ & $0.611(0.351-1.066)$ \\
\hline $2.0-2.9$ years $(n=1338)$ & 1.00 (reference) & $0.798(0.473-1.345)$ & $0.234(0.091$ to 0.605$)$ \\
\hline$\geq 3$ years $(n=2362)$ & 1.00 (reference) & $0.537(0.354$ to 0.815$)$ & $0.389(0.229$ to 0.661$)$ \\
\hline
\end{tabular}

OR = odds ratio; $95 \% \mathrm{CI}=95 \%$ confidence interval.

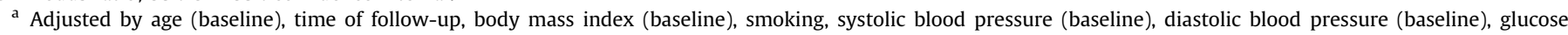

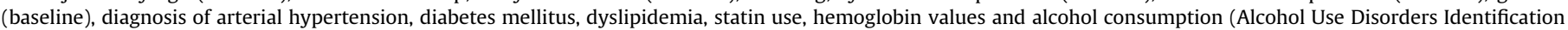
Test [AUDIT]).

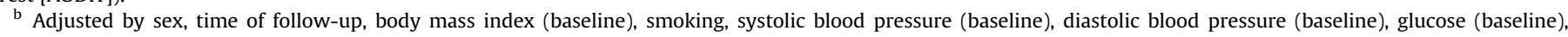

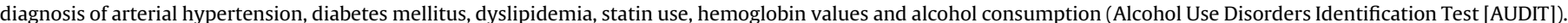

c Adjusted by age (baseline), sex, body mass index (baseline), smoking, systolic blood pressure (baseline), diastolic blood pressure (baseline), glucose (baseline), diagnosis of arterial hypertension, diabetes mellitus, dyslipidemia, statin use, hemoglobin values and alcohol consumption (Alcohol Use Disorders Identification Test [AUDIT]).

(both at baseline and follow-up) was associated with lower hsCRP values in both sexes (57\% and 60\% lower OR for men and women respectively). These associations were also observed across age groups, as well as in individuals with longer time of follow-up.

Table 5 shows the multivariate association of PAL with excess body weight and smoking status. Individuals who maintained high PAL with normal body mass index $\left(<25 \mathrm{~kg} / \mathrm{m}^{2}\right)$ had $71 \%$ less odds of having higher hsCRP ( $\mathrm{OR}=0.292$ [95\% CI: 0.110 to 0.774 ]), while physically active overweight/obese individuals had $57 \%$ less chance to have higher hsCRP (OR $=0.423$ [95\% CI: 0.284 to 0.630$]$ ).

\section{Discussion}

The main findings of the current study were: (i) physically active individuals (physically active at baseline and follow-up) had lower likelihood of having higher hsCRP; (ii) the maintenance of high PAL was associated with lower odds of higher hsCRP independent of sex, body mass index and smoking status; (iii) individuals who maintained PAL had decreased hsCRP values during the follow-up period (iv) longer maintenance of PAL was associated with greater decrease in hsCRP values.
Relatively small cross-sectional studies have shown an inverse relationship between PAL and hsCRP [14]. The results of the present study confirmed such a relationship between PAL and hsCRP, longitudinally, in a large sample. Interestingly, the association between PAL and hsCRP persisted after adjustment for BMI, which is closely associated with low-grade inflammation [15]. In addition, overweight and obese participants who maintained high PAL had $57.7 \%$ lower chances of having high levels of hsCRP, despite the changes in their BMI. Therefore, our results reinforce the important role that daily maintenance of PAL plays in the improvement of low-grade inflammation in adults with varied weight status.

In the current study, we demonstrated that the maintenance of PAL had a favorable impact on hsCRP values in both men and women, confirming the anti-inflammatory and cardio-protective effects of physical activity in both sexes. This observation is important because women are less physically active than men across their life span [16], presenting an increased risk of development of cardiovascular and metabolic diseases, particularly after menopause [17,18]. Interestingly, the impact of higher PAL on hsCRP values was more consistent with longer duration of follow-up visits, signifying the role of sustained maintenance of regular PAL

Table 5

Multivariate association between high hsCRP and physical activity levels according to excess body weight and smoking status ( $n=5030)$.

\begin{tabular}{|c|c|c|c|}
\hline \multirow[t]{3}{*}{ Variables } & \multicolumn{3}{|c|}{ Physically active (taking into account baseline and follow-up) } \\
\hline & None & Either baseline or follow-up & Both \\
\hline & OR $(95 \% \mathrm{CI})$ & OR $(95 \% \mathrm{CI})$ & OR $(95 \% \mathrm{CI})$ \\
\hline \multicolumn{4}{|l|}{ Body mass index $\geq 25 \mathrm{~kg} / \mathrm{m}^{2 a}$} \\
\hline None $(n=1669)$ & 1.00 (reference) & $0.671(0.350-1.286)$ & $0.292(0.110$ to 0.774$)$ \\
\hline Either baseline or follow-up $(n=607)$ & 1.00 (reference) & $2.190(0.777-6.175)$ & $1.195(0.349-4.088)$ \\
\hline Both $(\mathrm{n}=2754)$ & 1.00 (reference) & $0.690(0.508$ to 0.938$)$ & $0.423(0.284$ to 0.630$)$ \\
\hline \multicolumn{4}{|l|}{ Smoking (yes) } \\
\hline None $(\mathrm{n}=4453)$ & 1.00 (reference) & $0.693(0.518$ to 0.927$)$ & $0.437(0.300$ to 0.637$)$ \\
\hline Either baseline or follow-up $(n=298)$ & 1.00 (reference) & $1.481(0.462-4.753)$ & $0.938(0.228-3.857)$ \\
\hline Both $(n=279)$ & 1.00 (reference) & $1.120(0.445-2.818)$ & $0.113(0.023$ to 0.569$)$ \\
\hline
\end{tabular}

OR $=$ odds ratio; $95 \% \mathrm{CI}=95 \%$ confidence interval.

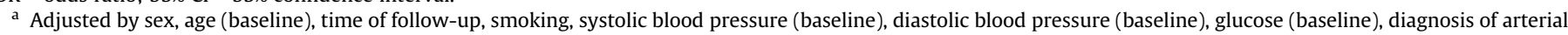
hypertension, diabetes mellitus, dyslipidemia, statin use, hemoglobin values and alcohol consumption (Alcohol Use Disorders Identification Test [AUDIT]).

b Adjusted by sex, age (baseline), time of follow-up, body mass index (baseline), systolic blood pressure (baseline), diastolic blood pressure (baseline), glucose (baseline),

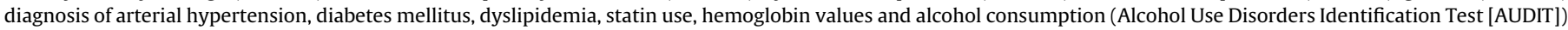


over longer periods for clinically relevant improvements in hsCRP values.

Maintenance of PAL was also associated with improvements in hsCRP values in all age categories, except for adults younger than 30 years-old. Loprizi et al. [19] in a large cross-sectional survey have also identified a negative relationship between objectively measured PAL and hsCRP values in adults ( $\geq 20$ years-old), but not in adolescents ( $\leq 19$ years-old). The underlying mechanisms, that explain this protective effect of PAL on low-grade inflammation only in subjects aged $>30$ years, are not clear. However, the progressive nature of cardiovascular risk including chronic low-grade inflammation may play a role, as in older ages the prevalence of these risk factors is higher.

The mechanisms that explain how PAL reduces inflammation and suppresses hsCRP values are not well defined. Acutely, contracting skeletal muscle produces and secretes several cytokines (myokines), most notably IL-6, which mediates metabolic changes during exercise [20]. IL-6 release from muscle increases up to 100fold during contractile exercise and its production results in increased systemic anti-inflammatory cytokines. Thus, it is possible that several expositions to exercise could lead to lower basal levels of circulating inflammatory markers, as well as reduce the inflammatory response to acute exercise. Moreover, other mechanism such as a decrease in cytokine production by adipose tissue, improvement in insulin sensitivity and improvement on endothelial function may lead lower levels of circulating inflammatory markers.

Limitations of this study should be recognized. The use of selfreported questionnaires to assess PAL, as opposed to more sophisticated objective approaches, is a limitation. However, notably, it has been previously demonstrated that the use of validated questionnaires from participants in their free-living conditions produced results similar to those when PAL was objectively measured [19]. In addition, despite the behavioral nature of physical activity, a reverse causality of inflammation causing inactivity cannot be discarded. Our study is also limited by the lack of information on the dose-response effect of PAL, thus precluding us from knowing the minimum threshold of PAL that is necessary to maintain optimum levels of hsCRP. The absence of more accurate measures of adiposity and other biochemical variables such as creatinine and uric acid limits additional interpretations that could have shed light on mechanisms. The number of participants with $<30 \mathrm{yrs}$ of age was relatively low and the potential influence of this limitation in the interpretation of the results cannot be discarded. Finally, we did not have data on menopausal status in women, which could be a potential confounding factor, as well.

In conclusion, the current study suggests that, in adults, the initiation of physical activity and the maintenance PAL play important roles in the attenuation of hSCRP levels, independently of sex, body weight and smoking status. The relationship between PAL and low-grade inflammation and the crucial role of maintenance of PAL in the reduction of hsCRP have profound clinical implications in the planning of preventive intervention strategies and physical activity-based education programs aimed at reducing the risk of atherosclerotic cardiovascular disease.

\section{Conflict of interest}

The authors declared they do not have anything to disclose regarding conflict of interest with respect to this manuscript.

\section{References}

[1] E. Paffen, M.P. DeMaat, C-reactive protein in atherosclerosis: a causal factor? Cardiovasc. Res. 71 (2006) 30-39.

[2] M.A. Mendall, D.P. Strachan, B.K. Butland, et al., C-reactive protein: relation to total mortality, cardiovascular mortality and cardiovascular risk factors in men, Eur. Heart J. 21 (2000) 1584-1590.

[3] P.M. Ridker, B.M. Everett, T. Thuren, et al., Antiinflammatory therapy with Canakinumab for atherosclerotic disease, N. Engl. J. Med. 377 (2017) 1119-1131.

[4] M.V. Fedewa, E.D. Hathaway, C.L. Ward-Ritacco, Effect of exercise training on C reactive protein: a systematic review and meta-analysis of randomised and non-randomised controlled trials, Br. J. Sports Med. 51 (2017) 670-676.

[5] A.S. Ribeiro, C.M. Tomeleri, M.F. Souza, et al., Effect of resistance training on Creactive protein, blood glucose and lipid profile in older women with differing levels of RT experience, Age 37 (2015) 109.

[6] C.M. Tomeleri, A.S. Ribeiro, M.F. Souza, et al., Resistance training improves inflammatory level, lipid and glycemic profiles in obese older women: a randomized controlled trial, Exp. Gerontol. 84 (2016) 80-87.

[7] A.M. Gerage, T.R. Benedetti, B.Q. Farah, et al., Sedentary behavior and light physical activity are associated with brachial and central blood pressure in hypertensive patients, PLoS One 10 (2015), e0146078.

[8] A.M. Gerage, R.M. Ritti-Dias, B. Balagopal, et al., Physical activity levels and hepatic steatosis: a longitudinal follow up study in adults, J. Gastroenterol. Hepatol. (2017 Aug 30), https://doi.org/10.1111/jgh.13965 [Epub ahead of print].

[9] R.M. Ritti-Dias, G.G. Cucato, W.L. do Prado, et al., Self-initiated changes in physical activity levels improve cardiometabolic profiles: a longitudinal follow-up study, Nutrition, metabolism, and cardiovascular diseases, Nutr. Metabol. Cardiovasc. Dis. 27 (2017) 48-53.

[10] P.H. Lee, D.J. Macfarlane, T.H. Lam, et al., Validity of the international physical activity questionnaire short form (IPAQ-SF): a systematic review, Int. J. Behav. Nutr. Phys. Activ. 8 (2011) 115.

[11] P.A. James, S. Oparil, B.L. Carter, et al., 2014 evidence-based guideline for the management of high blood pressure in adults: report from the panel members appointed to the Eighth Joint National Committee (JNC 8), Jama 311 (2014) 507-520.

[12] Cd Meneses-Gaya, A.W. Zuardi, S.R. Loureiro, et al., Alcohol Use Disorders Identification Test (AUDIT): an updated systematic review of psychometric properties, Psychology \& Neuroscience 2 (2009) 83-97.

[13] J.J. Cao, A.M. Arnold, T.A. Manolio, et al., Association of carotid artery intimamedia thickness, plaques, and C-reactive protein with future cardiovascular disease and all-cause mortality: the Cardiovascular Health Study, Circulation 116 (2007) 32-38.

[14] C. Kasapis, P.D. Thompson, The effects of physical activity on serum C-reactive protein and inflammatory markers: a systematic review, J. Am. Coll. Cardiol. 45 (2005) 1563-1569.

[15] K. Piestrzeniewicz, K. Luczak, J. Komorowski, et al., Relation of C-reactive protein to obesity, adipose tissue hormones and cardiovascular risk factors in men treated with early percutaneous intervention in course of acute myocardial infarction, Neuroendocrinol. Lett. 28 (2007) 427-432.

[16] S.J. McLaughlin, C.M. Connell, M.R. Janevic, Gender differences in trajectories of physical activity among older americans with diabetes, J. Aging Health 28 (2016) 460-480.

[17] S. Mayor, Early menopause is linked to higher risk of cardiovascular disease and death, review finds, Bmj 354 (2016) i5004.

[18] G. Mercuro, S. Zoncu, A. Cherchi, et al., Can menopause be considered an independent risk factor for cardiovascular disease? Italian heart journal official journal of the Italian Federation of Cardiology 2 (2001) 719-727.

[19] P. Loprinzi, B. Cardinal, C. Crespo, et al., Objectively measured physical activity and C-reactive protein: national health and nutrition examination survey 2003-2004, Scand. J. Med. Sci. Sports 23 (2013) 164-170.

[20] E.P. Plaisance, P.W. Grandjean, Physical activity and high-sensitivity C-reactive protein, Sports Med. 36 (2006) 443-458. 